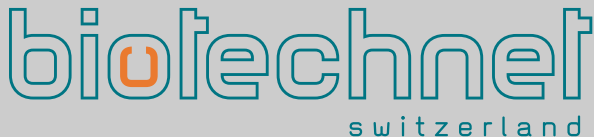

\section{D Bioprinted Muscle and Tendon Tissues for Drug Development}

Markus Rimanna, Sandra Laternsera, Hansjörg Keller ${ }^{b}$, Olivier Leupin ${ }^{b}$, and Ursula Graf-Hausner*a

${ }^{*}$ Correspondence: Prof. Dr. U. Graf-Hausnera , E-mail: guru@zhaw.ch alnstitute of Chemistry and Biological Chemistry (ICBC), Zurich University of

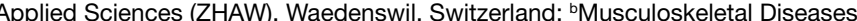
Novartis Institutes for BioMedical Research, Basel, Switzerland

Keywords: Alternative test method - Bioprinting standardization -3D cell culture $\cdot$ Drug development $\cdot$ Muscle-tendon disease $\cdot$ Specialized well plate

\section{Introduction}

In our aging societies, there is a huge medical need for treatments of degenerative muscle and tendon diseases, for which there are currently no approved pharmaceutical therapies. Furthermore, also devastating muscle diseases that affect children and younger patients such as Duchenne muscular dystrophy or amyothrophic lateral sclerosis (ALS) lack curative drug treatments. A major hurdle in new drug discovery and development is the nonexistence of normal functional human tissues and diseased tissues for compound screening and testing. Currently, most high-throughput drug screening campaigns are performed with target-centered biochemical or simple human cellbased assays if the drug target is known, or with two-dimensional (2D) cell culture phenotypic screens, if the target is unknown. Identified hits and further optimized compounds (leads) are then usually analyzed in low-throughput rodent ex vivo and/or in vivo preclinical animal models for efficacy, potency, specificity and safety. Besides the obvious slowness of this step to assess the pharmacodynamic and physiological effects of new drug candidates the jump from human cell-based systems to animal preclinical models and to human clinical trials is very often too large and not reliable enough to master. Fortunately, recent years have seen an incredible progress in new approaches generating functional 3D human tissues from normal and disease donors. Three-dimensional (3D) human organotypic tissue cultures are generally more predictive for in vivo effects, because they model much more in vivo tissue physiology than conventional 2D cell cultures. Thus, 3D tissue culture has the potential to revolutionize drug discovery and development. The paradigm shift from $2 \mathrm{D}$ to $3 \mathrm{D}$ cell culture is already showing great benefit in basic research of tissues differentiation and homeostasis as well as in tissue engineering efforts for regenerative medicine applications. ${ }^{[1]}$

Skeletal muscle and tendon tissues basically consist each of one single functional cell type: the multinucleated myotube and the tenocyte. Myotubes are the basic building block of skeletal muscle and are also called myofibers. They are formed by cell fusion of myoblasts. Myofibers are bundled into fascicles and an individual skeletal muscle can be made up of many fascicles surrounded by a connective tissue sheet. At each end, skeletal muscles are seamlessly and tightly connected to tendon through an interface called the myotendinous junction (MTJ). In contrast

\section{biotechnet Switzerland}

\author{
Hot from the press!
}

to skeletal muscle, which is mainly composed of cells with very little extracellular matrix (ECM), tendon tissue mainly consists of collagen ECM with few embedded threads of aligned cells. Over the last years, different groups have generated 3D skeletal muscle and tendon tissues for basic research and screening purposes. ${ }^{[2-5]}$ Tissues were generated between different kinds of attachment posts allowing mechanical tension and/or stimulation and functional readouts. However, although these systems allowed better in vitro investigations of skeletal muscle and tendon tissue generation and physiology as well as compound screenings they all suffer from a lack of reproducibility, robustness and cost effectiveness for high-throughput analyses and routine compound screenings.

\section{Novel 3D Bioprinted Human Mini-tissues for Compound Testing in a Specialized Well Plate}

The goal of the TEDD (Fig. 1) network project is to generate functional skeletal muscle and tendon mini-tissues by 3D printing (Fig. 2, bioprinter) in a novel 24-well plate system suitable for compound screening. Each well will be equipped with an insert containing two posts allowing tissue attachment and functional differentiation. A prototype insert with printed tissue attached to posts is schematically shown in Fig. 3. The system will be designed and developed in a way that electrical and/or mechanical stimulation and readouts of the tissues will be possible. Tissues are constructed by $3 \mathrm{D}$ bioprinting using a

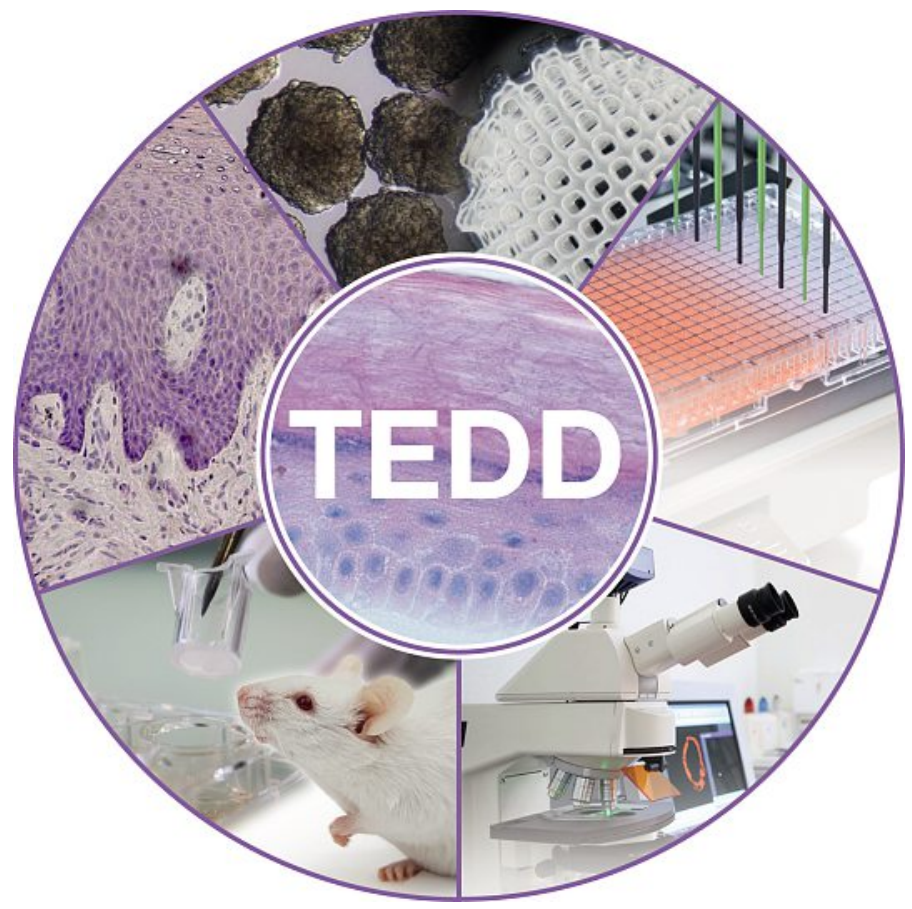

Fig. 1. TEDD is the Competence Centre for Drug Development and Substance Testing (www.icbc.zhaw.ch/tedd). TEDD is an incubator for network projects between industry and research departments at universities and applied universities to bring 3D cell culture technologies into routine applications. 


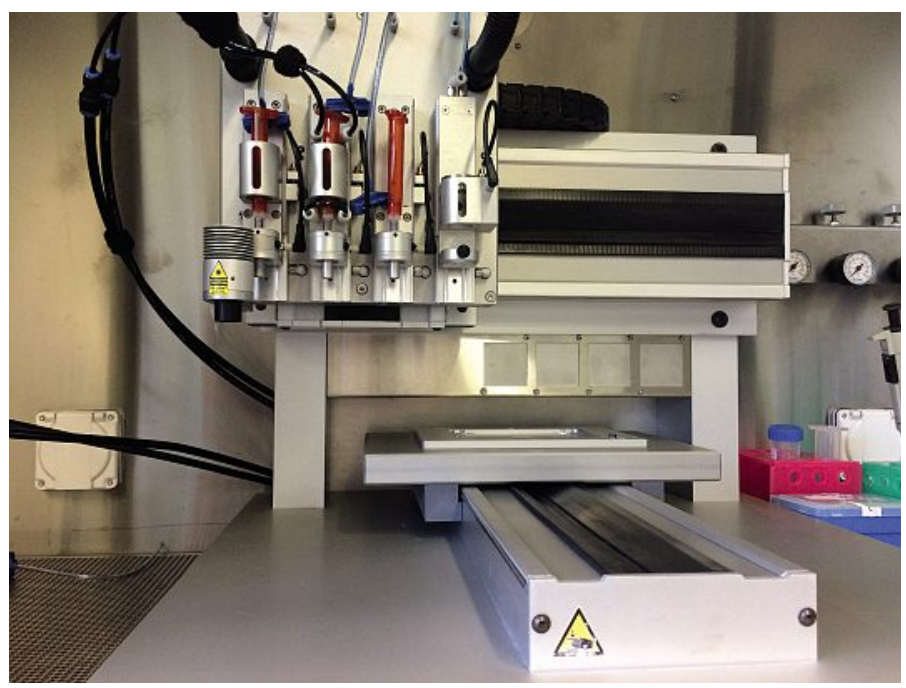

Fig. 2. Bioprinter 3DDiscovery from regenHU Ltd.

3D bioprinter (3DDiscovery, regenHU Ltd.). This allows precise spatially-resolved 3D tissue fabrication from well to well in a very reproducible and robust manner. The tissue is fabricated by printing alternating layers of matrix and cells. As matrix, a chemically-defined hydrogel called BioInk ${ }^{\mathrm{TM}}$ previously developed at $\mathrm{ZHAW}^{[6]}$ will be used to start with. Matrix layers are deposited by inkjet mode and contact printing with a needle in patterns defined by CAD software. Cells are jetted by inkjet mode printing onto matrix layers. The advantage of matrix and cell bioprinting in layers compared to standard 3D tissue generation by hydrogel encapsulation is the highly controlled spatial deposition of tissue components such as cells, biomaterial scaffolds and bioactive molecules. Especially in the production of 3D tissues harboring several different cell types the exact cell positioning and suitable microenvironment is crucial for proper tissue formation. We expect that the development of functional human mini-tissues using automated 3D bioprinting and specialized multiwell tissue culture labware for physiological readouts will pave the way for more efficient drug discovery and development, and early drug safety assessment. In addition, this approach has great potential to reduce the use of animals in pharma research thereby implementing the 3Rs (reduce, refine, replace) initiative.

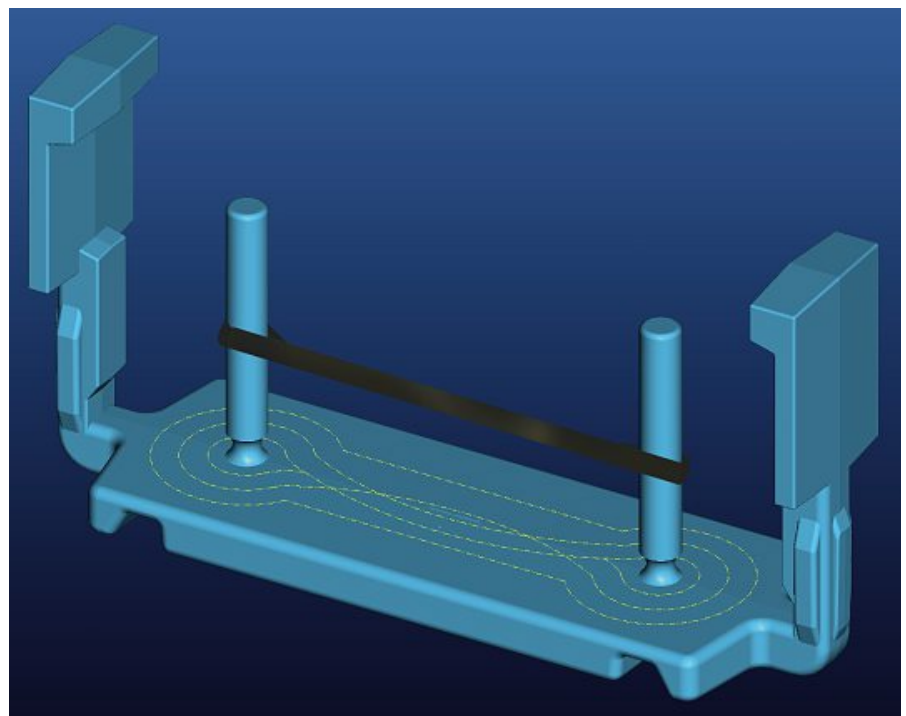

Fig. 3. Post design. Blue represents a possible post design that is clipped into a well of a 24-well plate. The brown structure between the posts shows suspended muscle/tendon tissue fibers.

\section{Preliminary Results}

Cells are printed using micro valve-based inkjet printheads. Therefore, the cyto-compatibility of the printing process for skeletal muscle and tendon cells was analyzed. Primary human skeletal muscle derived myoblasts and rat tenocytes, due to the current scarcity of healthy human tendon cells, were printed in alternating layers with BioInk ${ }^{\mathrm{TM}}$. A dumbbell-like form with holes at each of the ends was chosen to account for later printing around the two posts of the planned inserts. Viability staining (MTT) showed living human myoblasts of printed tissues after seven days in differentiation medium (Fig. 4A). Likewise, good viability was shown for bioprinted rat tenocytes after 11 days in culture as shown in Fig. 4B. Furthermore, the printing pattern of both cell types was still clearly visible. We analyzed differentiation of printed myoblasts into multinucleated myotubes during the seven-day differentiation period. Differentiation was assessed by immunostaining of the marker gene myosin heavy chain (MHC). Strong MHC staining was observed throughout the printed tissues indicating substantial presence of differentiated myotubes (Fig. 5A1). In a close up view, differentiated, multinucleated and cross-striated myofibers are shown (Fig. 5A2). As a surrogate marker of mature tendon tissue, the collagen I protein localization was analyzed. Fig. 5B1 shows bioprinted tendon tissue after 5 days of differentiation with the typical collagen I expression around the cell nuclei (Fig. 5B2). Additionally, qPCR marker gene expression analysis suggested extensive differentiation of myoblasts into myotubes and tenocytes into mature tendon (data not shown). Moreover, it demonstrated the biocompatibility of our BioInk ${ }^{\mathrm{TM}}$ with human skeletal muscle cell differentiation and tendon maturation.
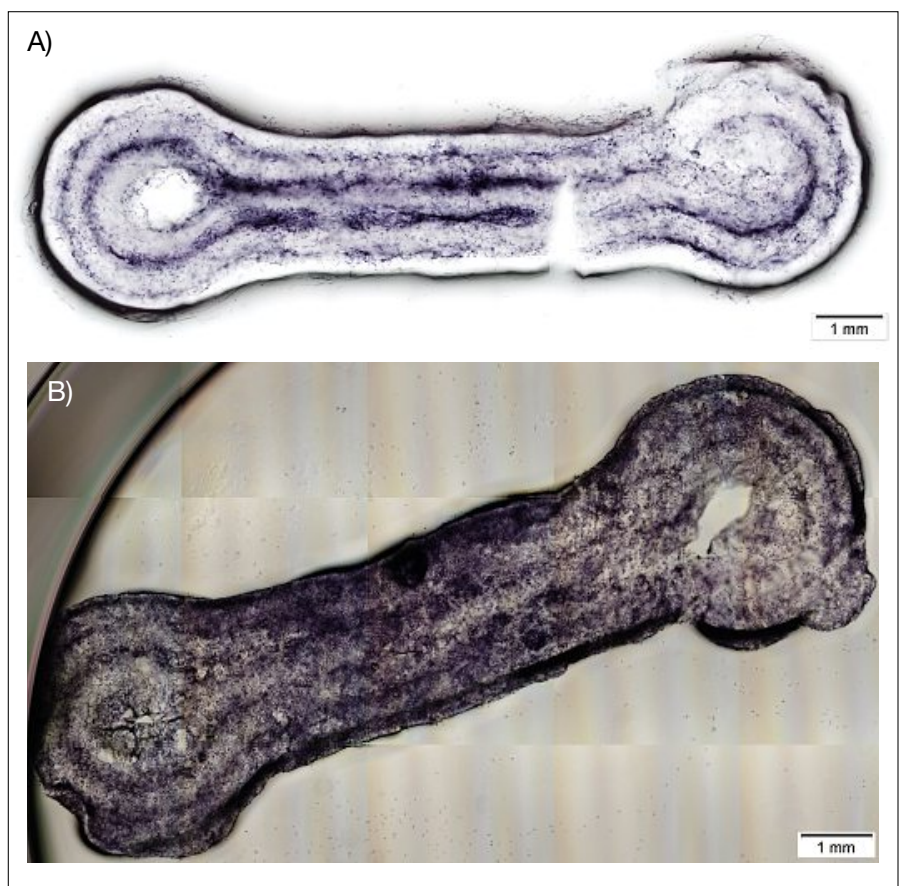

Fig. 4. Viability staining (MTT) of primary human myoblasts and rat tenocytes printed with Biolnk ${ }^{T M}$ in a layer-by-layer mode. A) Shows viable myoblasts after seven days in differentiation media. In B) viable tenocytes are shown at proliferation day 11.

\section{Outlook}

Tissue engineering by 3D bioprinting is gaining rapid momentum due to the accelerated development of specifically adapted 3D printers and a variety of cell-compatible biomaterial scaffolds as suitable printing matrices. The printing technology enables the precise deposition of bioactive molecules, cells 

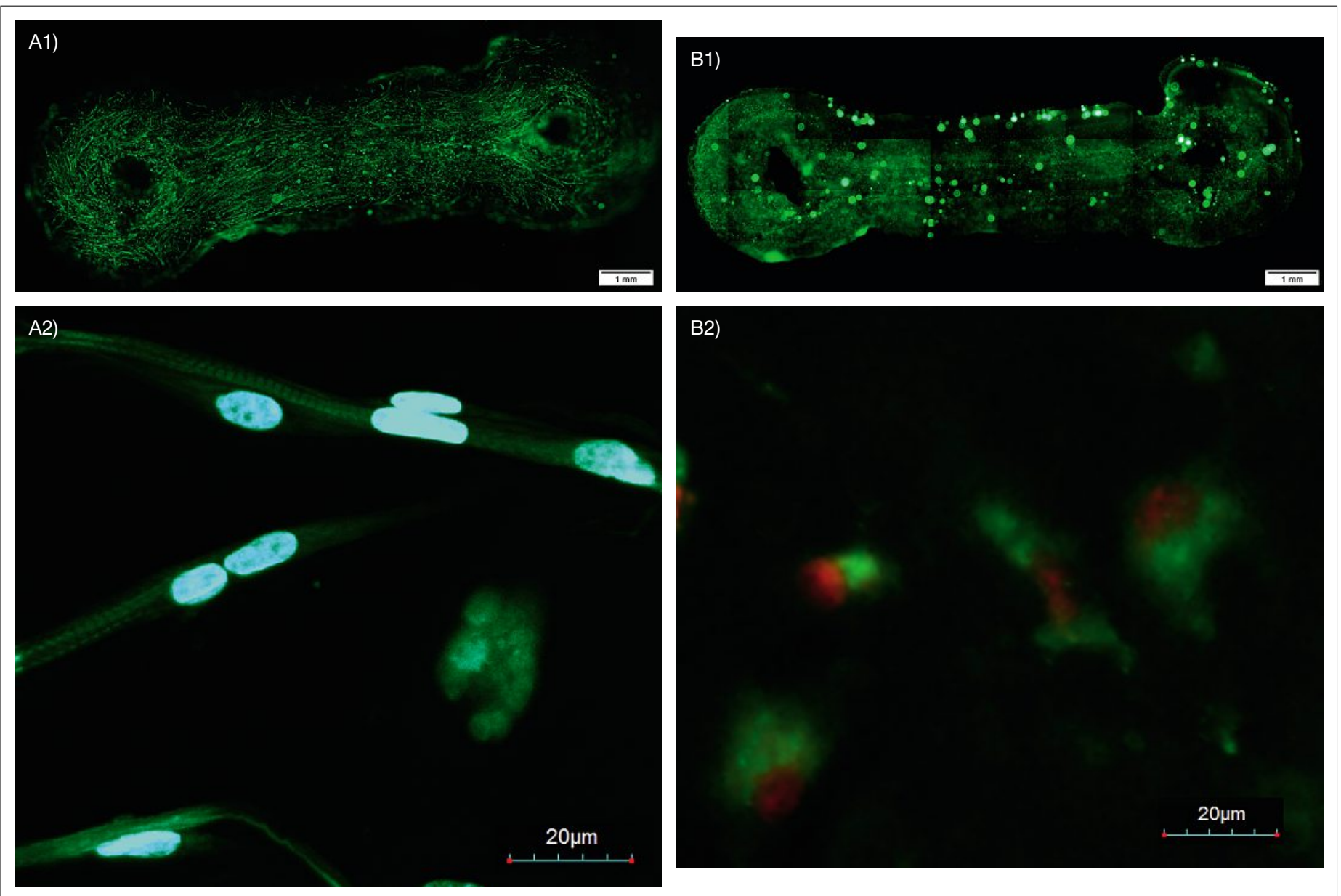

Fig. 5. Immunostaining of printed primary human myoblasts and rat tenocytes. In A) printed myoblasts were cultured for seven days in differentiation media and stained for myosin heavy chain (green). Characteristic muscle striation and multinucleated cell bodies are visible. A1 shows an overview picture of the entire printed structure. In B) printed tenocytes were cultured for five days in differentiation media and stained for collagen I (green). Characteristic mature tendon collagen I pattern around the cell nucleus is visible. B1 shows an overview picture of the entire printed structure. Nuclei stained with DAPI (blue) for muscle and with propidium iodide (red) for tendon tissue. A2 and B2 are close up views of A1 and B1, respectively.

and matrix components. This crucial advantage is going to revolutionize tissue engineering. As an example, it will allow us for the first time to generate and study the formation of the myotendinous junction between skeletal muscle and tendon in vitro. Furthermore, 3D bioprinting will enable the fabrication of normal human tissues and organotypic cultures from primary cells for basic research, pharmacological compound screening and drug safety testing. In addition, generation of human genetic and acquired disease tissues can be envisaged using ES/iPS cell technology and disease-inducing agents, respectively. Our project is also pioneering the combination of bioprinting technology with the development of sophisticated multiwell tissue culture plates for tissue stimulation and functional read-outs. Moreover, by merging these new tissue construction processes with state of the art micro-fluidic techniques it will be possible to culture engineered tissues under physiologic dynamic conditions. In the future, the 3D printing technology will not only provide 3D tissues but also the entire culture device to maintain cells under flow.

Besides applications in drug discovery, development and safety efforts, 3D bioprinting together with innovative large scale tissue culture will certainly also be groundbreaking for the fabrication of human tissue replacements in regenerative medicine. Thanks to this technology the current limitation of nutrient and oxygen supply of large tissues will be circumvented by the integration of printed blood vessels.

The bioprinting technology is still in its infancy. We are convinced that this technology shows a tremendous potential in tissue engineering and will pave the way to implement completely new production processes.

\section{Acknowledgements}

We gratefully thank the innovation promotion agency (CTI, Switzerland, Project No.: 16313.1) for financial support of the project and technical guidance. We thank the NTN Swiss Biotech for providing the platform to initiate such complex network projects between industry and applied research. Furthermore, we would like to thank our additional collaboration partners regenHU Ltd. and Weidmann Medical Technology AG for their contribution with the bioprinting technology and specialized labware production, respectively. Last but not least, we thank A. Alave, L. Hartmann, H. Jeker, and J. Richter for expert technical support and M. Rausch for imaging expertise.

Received: December 14, 2014

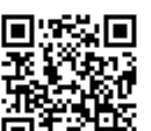

Please take a look at our new video describing our CTI cooperation.

[1] M. Y. Emmert, R. W. Hitchcock, S. P. Hoerstrup, Adv. Drug Deliv. Rev. 2014, 69, 254.

[2] Y. C. Huang, R. G. Dennis, L. Larkin, K. Baar, J. Appl. Physiol. 2005, 98, 706.

[3] H. Vandenburgh, Tissue Eng. Part B Rev. 2010, 16, 55

[4] M. Juhas, G. C. Engelmayr, Jr., A. N. Fontanella, G. M. Palmer, N. Bursac, Proc. Natl. Acad. Sci. USA 2014, 111, 5508.

[5] N. R. Martin, S. L. Passey, D. J. Player, A. Khodabukus, R. A. Ferguson, A. P. Sharples, V. Mudera, K. Baar, M. P. Lewis, Biomaterials 2013, 34, 5759.

[6] M. Rimann, E. Bono, H. Annaheim, M. Bleisch, U. Graf-Hausner, J. Lab. Automation 2015, doi:1177/221106814567146. 